\title{
RESEARCH
}

Open Access

\section{The effectiveness of PROLOTHERAPY for recalcitrant Medial TIBIAL Stress Syndrome: a prospective consecutive CASE series}

Nat Padhiar ${ }^{1,2,3^{*}} \mathbb{D}$, Mark Curtin ${ }^{1}$, Osama Aweid ${ }^{1}$, Bashaar Aweid ${ }^{1}$, Dylan Morrissey ${ }^{1}$, Otto Chan ${ }^{2}$, Peter Malliaras ${ }^{1,4}$ and Tom Crisp ${ }^{1,2}$

\begin{abstract}
Background: Medial tibial stress syndrome (MTSS) is one of the most common lower leg injuries in sporting populations. It accounts for between 6 and 16\% of all running injuries, and up to 53\% of lower leg injuries in military recruits. Various treatment modalities are available with varying degrees of success. In recalcitrant cases, surgery is often the only option.

Objective: To evaluate whether ultrasound-guided injection of 15\% dextrose for treatment of recalcitrant MTSS decreases pain and facilitates a return to desired activity levels for those who may otherwise be considering surgery or giving up the sport.

Method: The study design was a prospective consecutive case series involving eighteen patients: fifteen male and three female; (mean age $=31.2$ years) with recalcitrant MTSS. They were referred from sports injury clinics across the UK, having failed all available conservative treatment.

Intervention: An ultrasound-guided sub-periosteal injection of 15\% dextrose was administered by the same clinician (NP) along the length of the symptomatic area. Typically, $1 \mathrm{~mL}$ of solution was injected per $\mathrm{cm}$ of the symptomatic area.

Main outcome measures: Pain was assessed using a 10-cm visual analog scale (VAS) at baseline, short-term, medium-term (mean 18 weeks), and long-term (mean 52 weeks) follow-up. Symptom resolution and return to activity were measured using a Likert scale at medium and long-term follow-up. Statistical analyses were performed using SPSS for Mac version 19.0.0 (IBM, New York, NY, US). The Shapiro-Wilk test was used to evaluate the normality of the distribution of data. Friedman's non-parametric test was used to compare the within-patient treatment response over time. Post-hoc Wilcoxon signed-rank tests with Bonferroni corrections were performed to determine VAS average pain response to treatment over five paired periods.

(Continued on next page)
\end{abstract}

\footnotetext{
* Correspondence: nat.padhiar@londonsportswise.co.uk

${ }^{1}$ Centre for Sports and Exercise Medicine, William Harvey Research Institute,

Barts and The London School of Medicine and Dentistry, Queen Mary,

University of London, London, UK

${ }^{2}$ London SportsCare, London Independent Hospital, London, UK

Full list of author information is available at the end of the article
}

(c) The Author(s). 2021 Open Access This article is licensed under a Creative Commons Attribution 4.0 International License, which permits use, sharing, adaptation, distribution and reproduction in any medium or format, as long as you give appropriate credit to the original author(s) and the source, provide a link to the Creative Commons licence, and indicate if changes were made. The images or other third party material in this article are included in the article's Creative Commons licence, unless indicated otherwise in a credit line to the material. If material is not included in the article's Creative Commons licence and your intended use is not permitted by statutory regulation or exceeds the permitted use, you will need to obtain permission directly from the copyright holder. To view a copy of this licence, visit http://creativecommons.org/licenses/by/4.0/ The Creative Commons Public Domain Dedication waiver (http://creativecommons.org/publicdomain/zero/1.0/) applies to the data made available in this article, unless otherwise stated in a credit line to the data. 
(Continued from previous page)

Results: Patients reported a significant $(p<0.01)$ reduction in median VAS pain score at medium and long-term follow-up compared to baseline. Median improvement per patient was 4.5/10. Patients rated their condition as 'much improved' at medium-term follow-up and the median return to sports score was 'returned to desired but not pre-injury level' at medium-term and long-term follow-up. No adverse events were reported.

Conclusions: Ultrasound-guided 15\% dextrose prolotherapy injection has a significant medium-term effect on pain in MTSS. This benefit may be maintained long-term; however, more robust trials are required to validate these findings in the absence of controls.

Clinical relevance: Clinicians should consider the use of ultrasound-guided injection of 15\% dextrose as a viable treatment option to reduce pain and aid return to activity for patients with recalcitrant MTSS.

Keywords: Prolotherapy, MTSS, Injection, Exercise-induced leg pain, Dextrose

\section{Introduction}

MTSS is one of the most common lower leg injuries in sporting populations [1]. It accounts for between 6 and $16 \%$ of all running injuries [2], and up to $53 \%$ of lower leg injuries in military recruits [3].

The diagnosis is reliably based on a detailed history and physical examination with pain provoked on palpation of the lower one-third of medial tibial [4].

In the early stages of the condition, pain tends to occur at the beginning of exercise, may diminish as activity proceeds, and recurs at the end $[2,5,6]$. Usually, the pain resolves over a variable period of rest [7]. However, as the condition progresses, pain may occur constantly throughout the exercise [8], at rest, and at night, causing significant distress and affecting the quality of life $[2,5,9]$.

MTSS is predominantly managed conservatively. Treatment modalities that have shown potential benefit include extracorporeal shockwave therapy (ESWT), ultrasound therapy, iontophoresis, ice massage, periosteal pecking [10-13], non-steroidal anti-inflammatory drugs (NSAIDs), stretching and foot orthoses [14], and modification of biomechanical factors. However, a systematic review by Winters et al. [15] showed no evidence for the effect of any intervention in treating MTSS. In recalcitrant cases surgery is considered, although it significantly reduced pain in $72 \%$ of those treated, only $41 \%$ of patients returned to the sport at their previous level [16]. Although common, the pathophysiological process underlying MTSS remains uncertain. A popular theory is that excessive muscular traction could lead to inflammation of the periosteum, causing chronic periostitis [8, 17, 18]. However, histological studies have found scant evidence of inflammation [19-21]. More recently, it has been suggested that MTSS be classified as a point on a continuum of bone stress reaction, which can be assessed using magnetic resonance imaging (MRI) [22]. Beck (1998) proposed that repetitive loading during sustained weight-bearing activity may lead to strain-related periosteal remodeling due to tibial bending, which provokes stress injury at the point of maximum bending [23].

Winters et al. (2019) found linear microcracks in the biopsies of athletes with MTSS with no repair reaction, suggesting unrepaired microdamage as underlying pathophysiology [24].

Proliferative injection therapy (prolotherapy) has been used clinically since the late nineteenth century and has been mentioned in medical journals since at least 1937 [25]. The rationale behind prolotherapy is that injecting proliferants, such as hypertonic glucose solution, into damaged connective tissue, initiates inflammation, which leads to a healing cascade resulting in fibroplasia, deposition of new collagen and tissue hypertrophy [26] Animal studies have reported collagen proliferation, increased bone-ligament-bone junction strength and ligament mass with prolotherapy injections compared to controls [27]. The periosteum is richly innervated with nociceptive nerve fibers [28], therefore in MTSS, a prolotherapy injection may reduce pain by disrupting these sensory fibers as a result of the direct osmotic shock action of hypertonic dextrose on cells local to the injection site [26].

Trials of prolotherapy have found it to be beneficial in the treatment of lateral epicondylopathy [29], osteitis pubis [30], plantar fasciopathy [31], Achilles tendinopathy [32], and recalcitrant coccygodynia [33]. There is currently no published literature investigating the use of prolotherapy in the management of MTSS. A pilot study of this project reported a median pain reduction of $50 \%$ at medium-term follow-up [34]. The present study was a continuation of that pilot study and enabled longer-term treatment effects to be established. With current management options for recalcitrant MTSS showing inconsistent or unsatisfactory results, investigation of this novel treatment was necessary.

This prospective case series study sought to evaluate whether ultrasound-guided injection of hypertonic dextrose decreases pain and facilitates a return to desired activity levels for those who may otherwise be 
considering surgery or giving up the sport. We hypothesise that prolotherapy improves pain and facilitates a return to sport at the desired level.

\section{Patients and methods}

\section{Recruitment}

Sports physicians, orthopaedic surgeons, podiatrists, podiatric surgeons, and physiotherapists working in sports injury clinics who were known to the main 2 authors (NP, TAC) across the UK were contacted via email and invited to refer patients with painful, recalcitrant MTSS that had not responded to other conservative treatment modalities, whom they felt might benefit from the trial intervention (Fig. 1.). The failed conservative treatments included, (a) rest, ice, compression, elevation (RICE), (b) assessing and addressing any lower limb functional factors (muscle strength \& flexibility, proprioception \& balance, (c) therapeutic ultrasound therapy, (d) ESWT, (e) acupuncture, (f) needling, (g) Graston fascia release, (h) improving neurodynamic, (i) non-steroidal antiinflammatory drugs (NSAIDs), (j) correction of lower limb biomechanics (e.g., foot orthoses, brace, and taping for control of foot pronation), and (k) walking/running gait assessment and gait re-training.

Inclusion criteria were patients with persistent, painful MTSS assessed and confirmed by the lead clinician (NP). Exclusion criteria were previous periosteal surgery for MTSS, previous or current tibial stress fracture, or contraindications to the intervention such as pregnancy or anticoagulant therapy.

Patients were assessed by the lead clinician and the diagnosis of MTSS was confirmed by a history of exercise-induced pain over the posteromedial border of the middle to the distal third of the tibia, a positive Shin Palpation Test (palpation tenderness over the painful middle and distal thirds of the medial tibia) both at rest and following exercise, and MRI changes showing periosteal or bone marrow oedema.

Ethical approval was granted by the Queen Mary University of London Ethics Review Board (QMREC2009/ 22). Participants provided signed written informed consent. Subsequently, a detailed medical history and biographical data were taken and an examination was performed.

\section{Intervention and injection procedure}

The injection procedure is very simple and within the expertise of all clinicians who are used to injection techniques for musculoskeletal pathology. It is primarily performed in the out-patient department (OPD) and does not require a local anaesthetic even though local anaesthetic is used to dilute $50 \%$ glucose down to $15 \%$. In some cases where pain tolerance is poor, it can be performed under a general anaesthetic. In this study, all subjects were treated in OPD. The target area for needle placement in the area anterior to the deep crural fascia (Figs. 2 and 3.) along the medial tibia. The skin overlying the most painful area of the tibia was marked with an indelible marker pen and then cleansed using alcoholic chlorhexidine (2\% chlorhexidine gluconate, $70 \%$ isopropyl alcohol). An ultrasound scanner (USS) (Siemens AG, Berlin, Germany) was used to guide and confirm needle position. The needle is introduced under real-time USS from the most proximal end of the site of pain (knee end) to the most distal (ankle end) (Figs. $4 \mathrm{a} \& \mathrm{~b}$ ). The spinal needle $(0.7 \mathrm{~mm}$ diameter $\times 90 \mathrm{~mm}$ length, Becton, Dickinson and Company LLC, Franklin Lakes, New Jersey, US) was positioned parallel along the medial tibia in the area anterior to the deep crural fascia (Fig. 5). The needle introducer was removed with the needle in place. $15 \%$ dextrose solution was slowly infiltrated, approximately $1 \mathrm{~mL}$ of solution per $1 \mathrm{~cm}$ along the whole length of the area of pain. In some cases where the length was longer than the spinal needle, the second entry point was made following the same protocol as above. After the injection, the area was cleaned, dressed, ice packs applied for $2 \mathrm{~min}$, and knee high compression socks (Fig. 6.) (Bauerfeind AG, Zeulenroda-Triebes, Germany) were fitted. Patients were advised to continue wearing them for up to four weeks, removing them at night. Patients were advised to take relative rest for three days and advised for simple flexibility exercises and a graded return to physical activity. Patients were followed up one week later to monitor progress and address any concerns or questions. Patients were also given an emergency mobile number to call if they experienced any adverse reaction to the injection. This would include, extreme unrelenting pain, erythema, swelling, delayed allergic rash or sleep disturbance.

\section{Outcome measures}

The patients' 'average pain', defined as the most pervasive severity of pain throughout $24 \mathrm{~h}$, was measured using a $10-\mathrm{cm}$ visual analog scale (VAS). The VAS is sensitive [35], reliable, valid, and responsive for measuring pain in other common musculoskeletal conditions, such as patellofemoral pain syndrome [36]. The pain was assessed in this way at 0 (baseline), 1, 2, and 4 weeks after the injection, and at medium-term (mean 18 weeks, range 13-36 weeks) and long-term (mean 52 weeks, range 47-74 weeks) follow-up to assess the patients' response to the intervention.

A Likert [37] symptom resolution scale was used to measure the subjective degree of recovery at medium-term and long-term follow-up compared to baseline. There are six possible outcome scores for 
18 Patients (15 male, 3 females, mean age 31.2 years) with recalcitrant MTSS accepted the invitation.

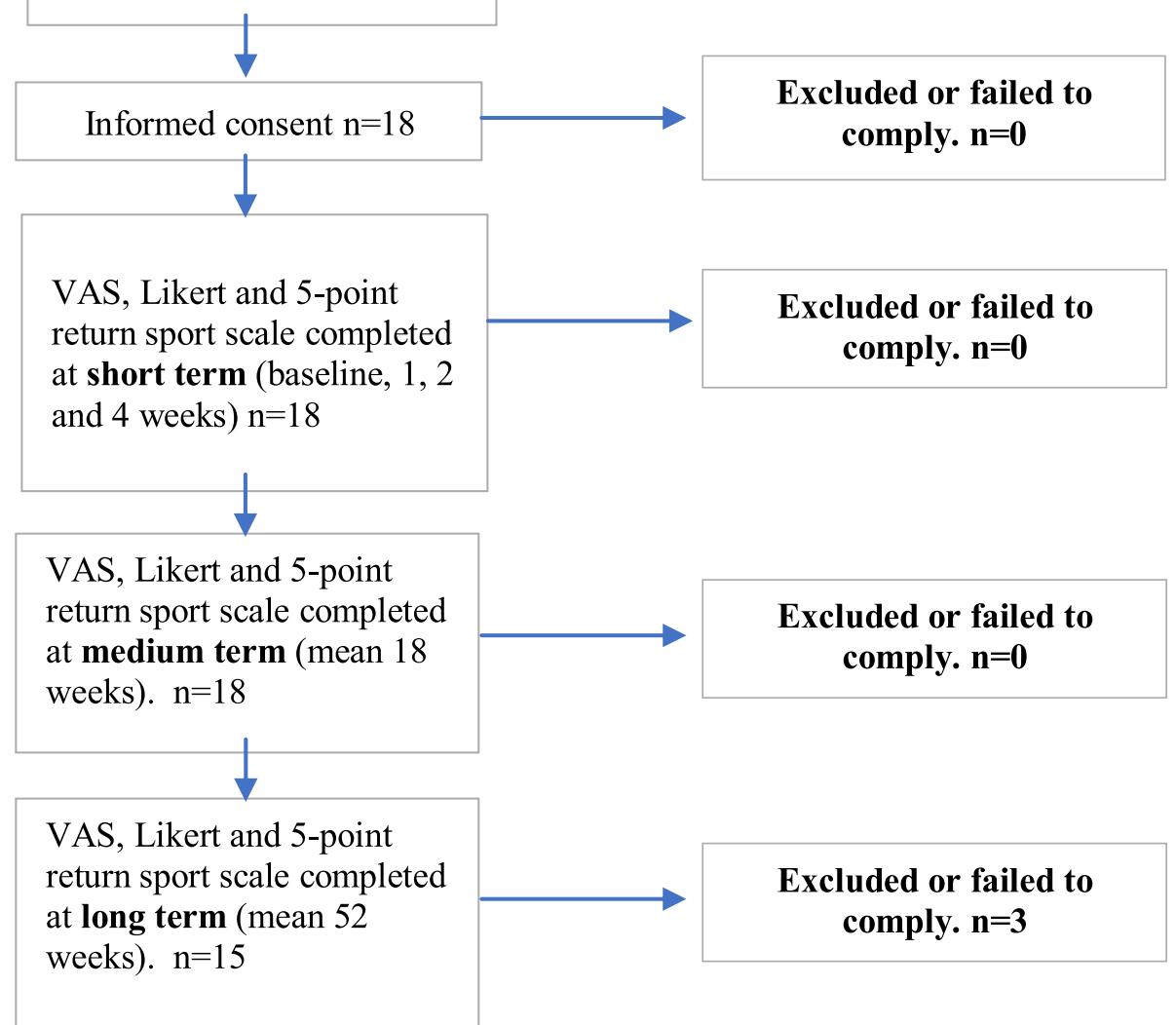

Fig. 1 Recruitment process

the Likert scale: $1-$ completely recovered, 2- much improved, 3- somewhat improved, 4- no change, 5worse, 6- much worse. Treatment was classed as a success in patients who rated themselves as 'completely recovered' or 'much improved', reflecting the method of previous authors [38]. Other scores were considered a treatment failure. Categorical scales such as the Likert are sensitive indicators of clinical trial endpoints [37].

Return to the sport was assessed with a five-point activity scale at medium-term and long-term followup: 1- not active at all, 2- no return to sport, 3returned to the sport at an unsatisfactory lower level, 4- returned at desired but not pre-injury level, 5returned at the pre-injury level. Although not reported in other literature, treatment aimed to enable patients to return to their desired sports at pre-injury levels. All other activity scores were considered a treatment failure.

\section{Statistical analysis}

Statistical analyses were performed using SPSS for Mac version 19.0.0 (IBM, New York, NY, US). Statistical significance was set at a $p$-value less than 0.05 . The ShapiroWilk test was used to evaluate the normality of the distribution of data. The distribution of data were negatively skewed and not normally distributed (Shapiro-Wilk = 0.009) therefore appropriate non-parametric tests were performed to evaluate the changes in pain levels.

Median values and interquartile ranges were calculated to compare baseline and follow-up data for VAS average pain scores, Likert symptom resolution, and return to sports scores. 


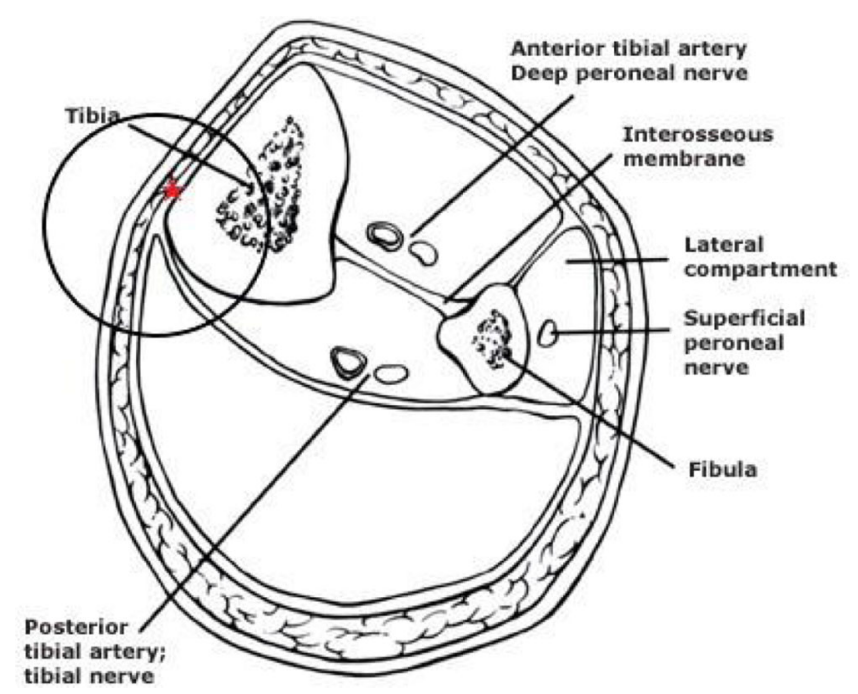

Fig. 2 Cross section diagram of the leg. Red star marks the target area, anterior to deep crural fascia along the medial tibia

Friedman's non-parametric test was used to compare the within-patient treatment response over time. Post-hoc Wilcoxon signed-rank tests with Bonferroni corrections were performed to determine VAS average pain response to treatment over five paired periods (baseline - 4 weeks, baseline - 18 weeks, 4-18 weeks, baseline - 52 weeks, 4 weeks 52 weeks). The Bonferroni corrected alpha value was $(p<0.01)$.

\section{Results}

Twenty-five legs of eighteen patients were injected, of that twenty-five, seven legs were injected a second time. For data collection, each patient was treated as a whole case rather than individual legs.
On average, patients reported that post-injection pain took three days to settle. Side effects were not asked for specifically, but there were no self-reported adverse events following injection.

The mean age and duration of symptoms for the patients are summarised in Table 1. Two patients had undergone surgical fasciotomy for diagnosed chronic compartment syndrome on their affected limbs.

\section{Descriptive analysis}

Data for eighteen patients were available for analysis of treatment effect to medium-term and fifteen patients to long-term follow-up. Three patients were lost to followup and these data points were not imputed but omitted from the long-term analysis.

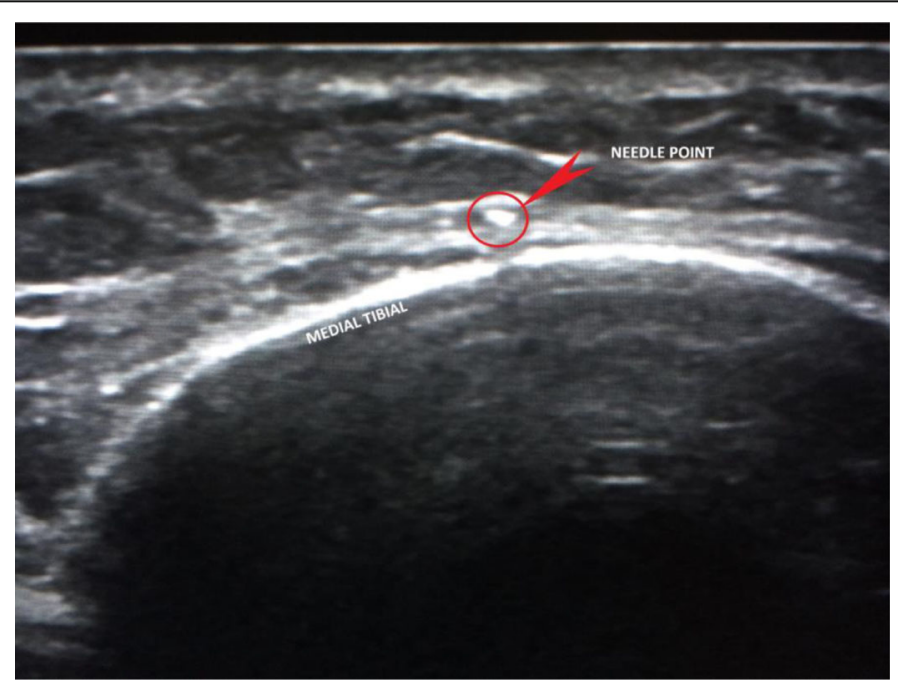

Fig. 3 Transverse ultrasound image of the needle position (marked with the red circle/arrow) 
A. The spinal needle was inserted under ultrasound guidance into the medial tibia and just anterior to start of the deep crural fascia region under ultrasound guidance.

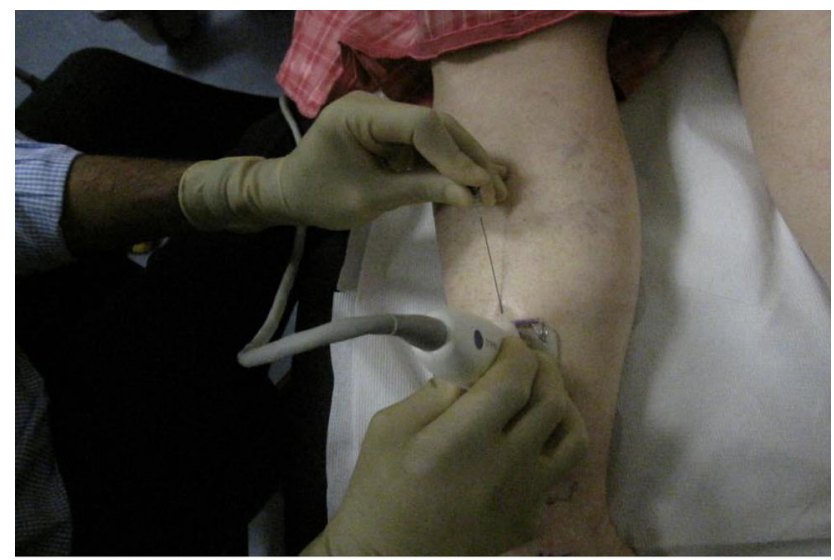

B. Longitudinal USS image showing the needle position. Please note that initially it is an angle but final position needs to be parallel with the medial tibia at the target site.

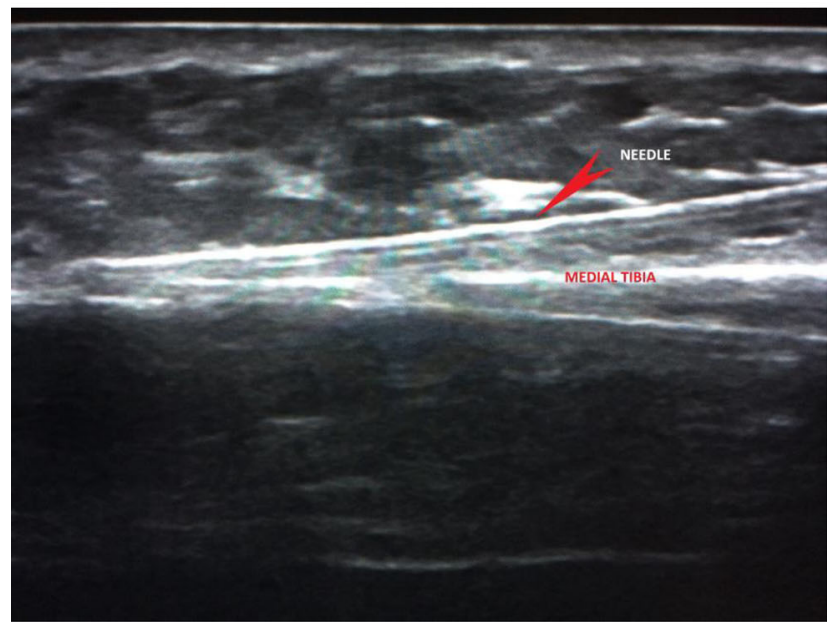

Fig. 4 A. The spinal needle was inserted under ultrasound guidance into the medial tibia and just anterior to start of the deep crural fascia region under ultrasound guidance. $\mathbf{B}$. Longitudinal USS image showing the needle position. Please note that initially it is at an angle but final position needs to be parallel with the medial tibia at the target site

Medians and interquartile ranges for the three outcome measures are displayed in Table.2. Change in median VAS average pain score over time is displayed in Fig. 7.

There was complete resolution of symptoms at 18 weeks and 52 weeks in two patients (11\%). There were much improved symptoms at 18 weeks in eight patients (33\%) and 52 weeks in three patients $(20 \%)$. At 18 weeks, six patients (33\%) were somewhat improved and at 52 weeks the same improvement was observed in three patients (20\%). There was no change in symptom resolution at 18 weeks in two patients (11\%) and 52 weeks in seven patients (47\%). There were no patients who were worse or made worse following prolotherapy (Table.3.).
At 18 weeks, five patients (28\%) returned to preinjury level return to sport, and at 52 weeks four patients (27\%). At 18 weeks, five patients (28\%) returned to the desired but not pre-injury level return to sport, and at 52 weeks, four patients (27\%). At 18 weeks, eight patients (44\%) returned to the sport at an unsatisfactory level, and at 52 weeks, six patients (40\%). One patient failed to return to any level of sport or activity and elected to undergo surgery (Table.3.).

\section{Statistical analysis}

Friedman's test of the VAS average pain scores to medium-term (mean 18 weeks) reported a Chi-squared value of $30.3, p<0.001$. There was a statistically 


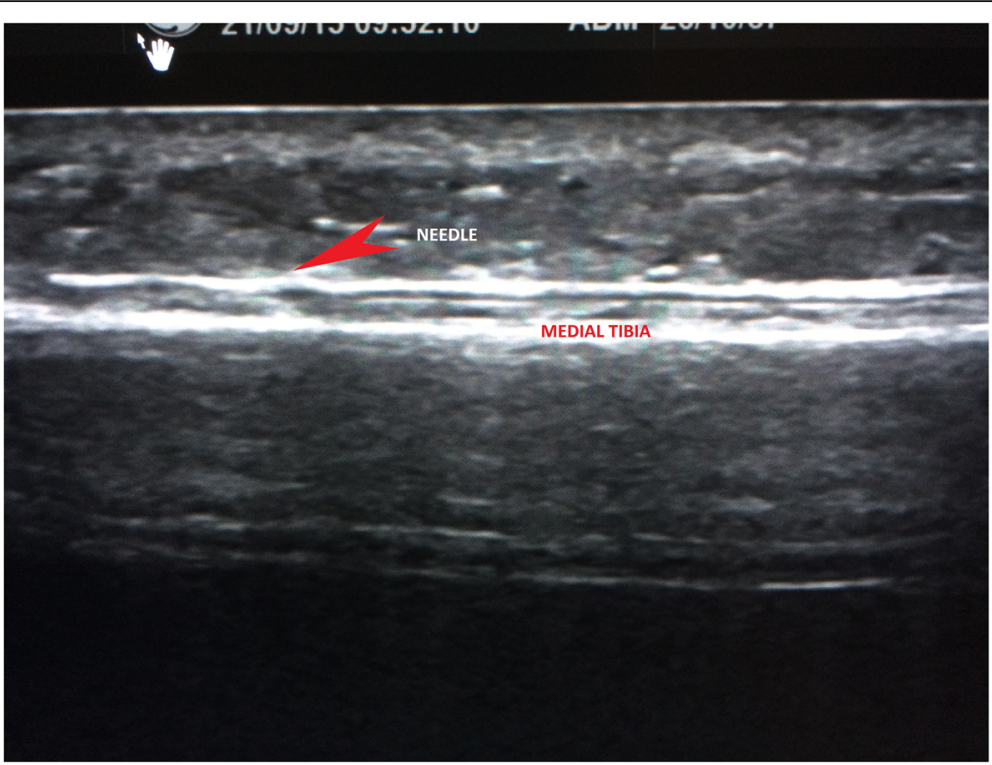

Fig. 5 Longitudinal USS image showing the final needle position which is positioned parallel with the medial tibia at the target site

significant difference between the mean pain ranks over time. Therefore, a post-hoc analysis was warranted. Friedman's test was also significant to long-term followup, reporting a Chi-squared value of 27.5, $p=<0.001$.

Changes in VAS average pain score rank and $p$-values for the five paired periods are reported in Table 4. VAS average pain decreased for 16 patients from baseline to both 4 weeks $(p<0.001)$ and 18 weeks follow-up $(p<$ 0.001 ). One patient had an increase in pain at 4 weeks which subsequently decreased at 18 weeks follow-up and another patient reported no change at 4 weeks and 18 weeks. Between 4 weeks and 18 weeks after the injection, the pain increased in seven patients (38.8\%), decreased in five (27.7\%), and remained the same in six patients (33.3\%) $(p=0.405)$. Between baseline and 52 weeks, pain decreased in 13 patients $(86.7 \%)$, remained the same in one patient $(6.7 \%)$, and increased in one patient $(6.7 \%)$ $(p=0.001)$. Between 4 weeks and 52 weeks, pain decreased in five patients (33.3\%), remained the same in four patients $(26.7 \%)$, and increased in six patients $(40 \%)$ $(p=0.322)$.

After Bonferroni corrections were applied, the change in VAS average pain from baseline to 4,18 , and 52 weeks follow-up were statistically significant $(p<0.01)$. The changes in VAS average pain from 4 to 18 weeks and 4 to 52 weeks were not significant $(p>0.01)$.

\section{Discussion}

The pain was significantly reduced $(\mathrm{p}<0.01)$ over short, medium, and long-term compared to baseline, with only two patients not reporting a reduction in VAS average pain over these periods (Table 4). The median VAS average pain score improved by 4.5 points at medium and long-term follow-up compared to baseline, equivalent to a $60 \%$ reduction in pain (Table 2.).

However, a wider range of pain scores was seen in the long-term (Fig. 5.), in addition to a larger $p$-value for average pain reduction, possibly due to the lower statistical power at this stage of follow-up. There was also a trend for improvement in pain to recede from 4 weeks post-injection, suggesting that pain control is most effective within the first month after administration. This is a potential window of opportunity to implement other conservative management options that could facilitate long-term pain control.

At 18 weeks (medium-term) follow-up, ten patients (55.5\%) reported their MTSS as 'completely recovered' or 'much improved', indicating treatment success at this stage. Six patients (40\%) fulfilled these criteria at 52 weeks follow-up, which signifies a potential longer-term decline in treatment effect (Table 4.).

Five patients $(27.7 \%)$ reported a return to sport at preinjury levels at medium-term follow-up and four patients $(26.7 \%)$ at long-term follow-up. Treatment seems to have a limited effect on this outcome measure. However, ten patients $(55.5 \%)$ were active at the desired level or more at medium-term follow-up and eight (53.3\%) at long-term follow-up, which suggests we may have been too stringent in selecting the treatment success criteria for this outcome measure. One patient elected to undergo surgery, as at 52 weeks there was no improvement and failed to return to sport.

\section{Conclusion}

Dextrose prolotherapy injection was well tolerated and significantly improved pain in the short term and, its 


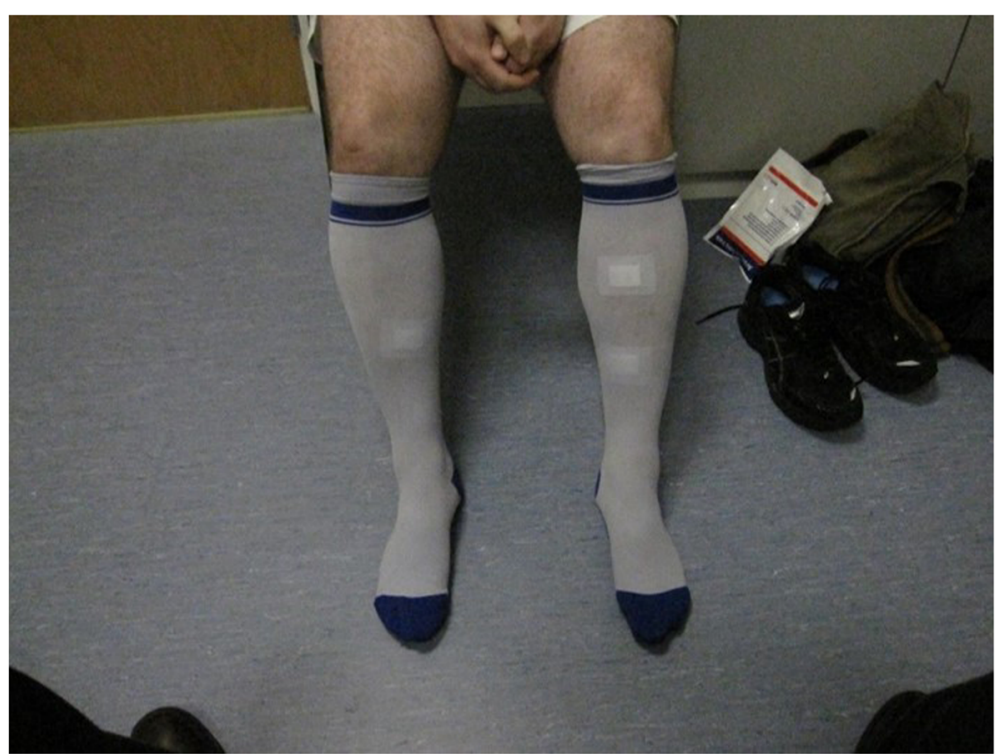

Fig. 6 After the injection care involved cleansing the skin, applying wound dressing, ice and compression socks

effect was retained in the long term. Adequate symptom resolution and return to sport were achieved at medium-term follow-up and return to sport were maintained long-term. This prospective consecutive case study consisting of selective recalcitrant cases, therefore, suggests that prolotherapy has a significant effect on short, medium, and long-term pain reduction but in the absence of controls, a more robust study is required to show this benefit.

Prolotherapy is an effective treatment modality in the management of recalcitrant MTSS and even though one patient elected to undergo surgery, this study was not designed to assess whether there will be a reduction in the number of patients that may require surgery. This study did not involve any histological sampling to explain the effect on the tissue. We postulate that $15 \%$ glucose acts as an osmotic and chemical irritant (dehydrating cells) along with damage to tissue through needling causing local trauma and bleeding. This provokes a cascade of inflammation, proliferation, and remodeling. The rationale behind prolotherapy is that injecting proliferants, such as hypertonic glucose solution, into damaged connective tissue, initiates

Table 1 Mean age and duration of symptoms

\begin{tabular}{ll}
\hline Characteristic & \\
\hline Male & $\mathbf{1 5}(\mathbf{8 3 \% )}$ \\
\hline Female & $3(17 \%)$ \\
Mean age & 34 (SD 10.7) \\
Mean symptom duration (weeks) & 52 (SD 9.1) \\
Mean BMl & 25.9 (SD 3.4) \\
\hline
\end{tabular}

BMI = Body Mass Index inflammation, which leads to a healing cascade resulting in fibroplasia, deposition of new collagen, and tissue hypertrophy [26]. It is also possible that prolotherapy improves the mechanical advantage with improved stability of deep crural fascia junction with the medial tibia. Animal studies have reported collagen proliferation, increased bone-ligament-bone junction strength, and ligament mass with prolotherapy injections compared to controls [27]. The reduction in pain may be due periosteum being richly innervated with nociceptive nerve fibers [28], therefore, in MTSS a prolotherapy injection may reduce pain by disrupting these sensory fibers as a result of the direct osmotic shock action of hypertonic dextrose on cells local to the injection site [26]. It is also worth debating, even though there is no evidence, whether and if, the sensory fibers are disrupted, could this, apart from the positive effects on pain and function short-term, produce adverse effects long term e.g., disturbed bone remodeling potentially leading to stress fractures/worsening MTSS/osteoporosis?

However, in sport, the benefit of a pain-free phase can be effectively used to provide a platform for early rehabilitation and a window of opportunity to plan a return to sport or physical activity-specific rehabilitation.

\section{Limitations}

As the first study investigating this novel injection technique, the use of a non-blinded, consecutive case series design without randomisation or a control group was a pragmatic choice given the difficulties of recruiting participants. Evidence of treatment effect is therefore limited. Despite results showing a significant treatment effect on pain, the mechanism by which it exerts this 
Table 2 Median values and interquartile ranges for VAS average pain, symptom resolution and level of activity

\begin{tabular}{|c|c|c|c|}
\hline & & Median & Interquartile range \\
\hline \multirow{5}{*}{$\begin{array}{l}\text { VAS average pain score }(\mathbf{c m}) \\
0-\text { no pain } \\
10-\text { worst pain imaginable }\end{array}$} & Baseline & 7.5 & $6-8$ \\
\hline & 2 weeks & 2 & $1-3.75$ \\
\hline & 4 weeks & 3 & $1-4$ \\
\hline & 18 weeks & 3 & $2-4$ \\
\hline & 52 weeks & 3 & $2-4.5$ \\
\hline \multirow{2}{*}{$\begin{array}{l}\text { Likert symptom resolution score } \\
1 \text { - completely recovered } \\
6 \text { - much worse }\end{array}$} & 18 weeks & 2 & $2-3$ \\
\hline & 52 weeks & 3 & $2-4$ \\
\hline \multirow{2}{*}{$\begin{array}{l}\text { Activity level } \\
1-\text { not active at all } \\
5 \text { - active at pre-injury level } \\
\end{array}$} & 18 weeks & 4 & $3-5$ \\
\hline & 52 weeks & 4 & $3-4.5$ \\
\hline
\end{tabular}

effect cannot be determined, and therefore the possibility of a placebo effect cannot be excluded in addition to the possibility these patients may have improved with time without treatment.

Patients were only included in the study if they had MRI evidence of periosteal changes or bone marrow oedema. MTSS is a clinical pain syndrome with unknown aetiology. The evidence suggests that periosteal and bone marrow oedema are absent in the majority (i.e., 56\%) of clinically diagnosed cases with MTSS [39]. Furthermore, periosteal and bone marrow oedema is often present in healthy asymptomatic athletes $[40,41]$ which suggests it is not an adequate characteristic to identify those with MTSS. As a result, we may have missed relevant cases with MTSS with negative MRI findings.

Three patients were also lost to long term follow up, despite every effort to contact them to complete the study. In a consecutive case series of 18 subjects, this was a great loss.

Given that there were no validated scales to specifically assess treatment outcomes in EILP patients at the time of this study, we adapted Likert scales to measure symptom resolution and return to sport. Although Likert scales are sensitive indicators of clinical trial endpoints

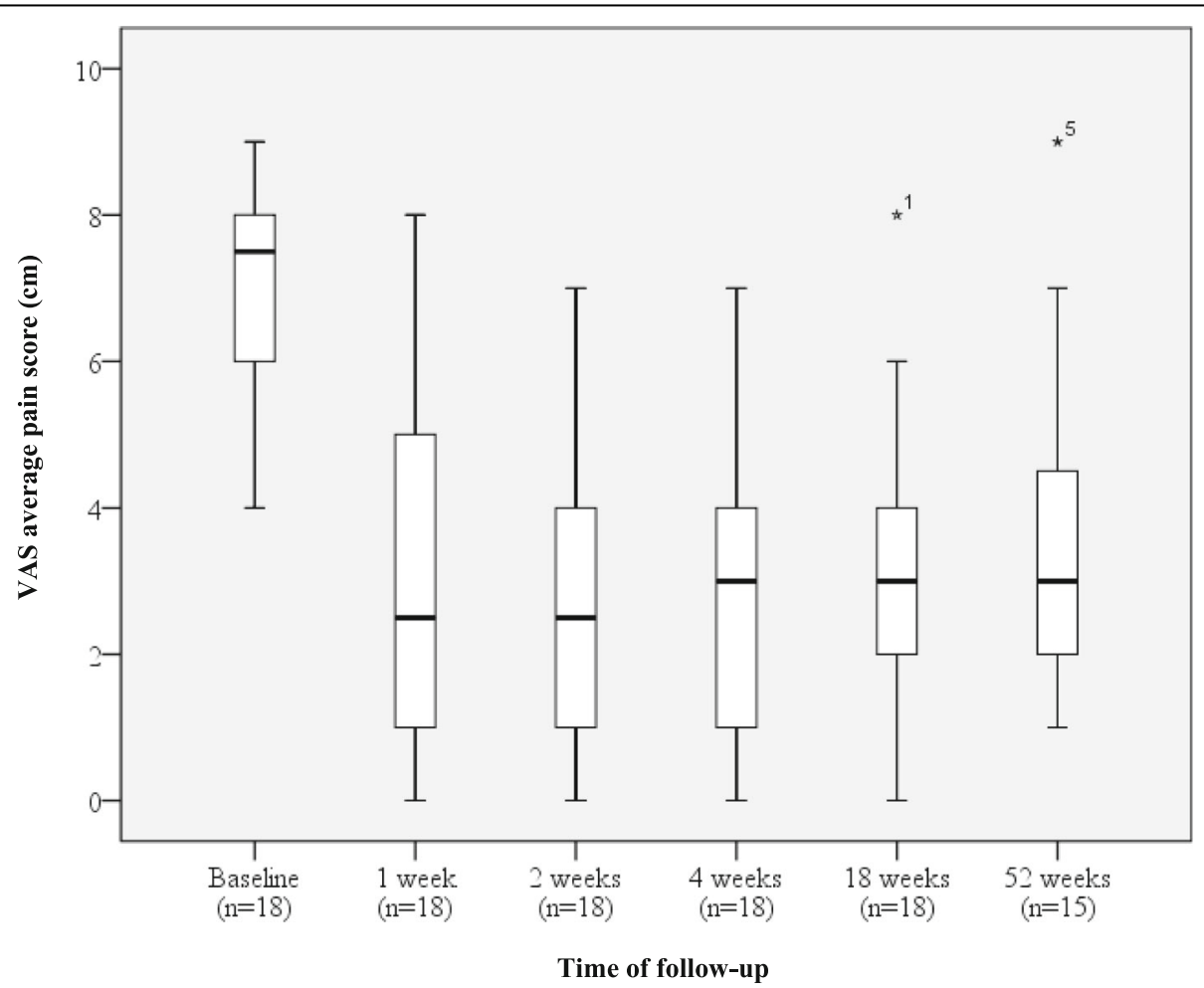

Fig. 7 Box plot of median and interquartile range VAS average pain scores for the group of patients at follow-up. ${ }^{*}$ Indicates a potential outlier (number refers to patient number) 
Table 3 Summary of symptom resolution and activity level outcomes

\begin{tabular}{lll}
\hline & $\mathbf{1 8}$ weeks $(\boldsymbol{n}=\mathbf{1 8})$ & $\mathbf{1}$ year $(\boldsymbol{n}=\mathbf{1 5})$ \\
\hline $\begin{array}{l}\text { Likert symptom resolution } \\
\text { Completely recovered }\end{array}$ & $2(11.11 \%)$ & $2(13.33 \%)$ \\
Much improved & $8(33.33 \%)$ & $3(20 \%)$ \\
Somewhat improved & $6(33.33 \%)$ & $3(20 \%)$ \\
No change & $2(11.11 \%)$ & $7(46.67 \%)$ \\
Worse & $0(0 \%)$ & $0(0 \%)$ \\
Much worse & $0(0 \%)$ & $0(0 \%)$ \\
Activity level & & $4(26.67 \%)$ \\
Returned at pre-injury level & $5(27.78 \%)$ & $4(26.67 \%)$ \\
Returned at desired but not pre-injury level & $5(27.78 \%)$ & $6(40 \%)$ \\
Returned to sport at an unsatisfactory lower level & $8(44.44 \%)$ & $1(6.67 \%)$ \\
No return to sport & $0(0 \%)$ & $0(0 \%)$ \\
Not active at all & $0(0 \%)$ & \\
\hline
\end{tabular}

[37], the lack of specificity could at least partly explain the limited treatment effect for these outcomes. Since this study was conducted there are 3 validated patient outcome scores are now available [42-44], one is specific for MTSS [42].

\section{Context}

The current literature on the effects of prolotherapy on chronic musculoskeletal conditions (lateral epicondylopathy, Achilles and other tendinopathies, osteitis pubis, plantar fasciopathy, recalcitrant coccygodynia, and

Table 4 Rank data and significance for the Wilcoxon signedrank tests for VAS average pain. A negative rank represents an improvement in a patient's pain over that time period. A positive rank represents worsening pain over that time period

\begin{tabular}{llll}
\hline Time period & Ranks & N & p-value \\
\hline $\begin{array}{l}\text { Baseline } \mathbf{- 4} \text { weeks } \\
(n=18)\end{array}$ & Negative ranks & 16 & $<0.001$ \\
& Positive ranks & 1 & \\
Baseline - 18 weeks & Ties & 1 & \\
$(n=18)$ & Negative ranks & 16 & $<0.001$ \\
& Positive ranks & 0 & \\
$\mathbf{4}$ weeks $-\mathbf{1 8}$ weeks & Ties & 2 & \\
$(n=18)$ & Negative ranks & 5 & 0.405 \\
& Positive ranks & 7 & \\
Baseline $-\mathbf{5 2}$ weeks & Ties & 6 & \\
$(n=15)$ & Negative ranks & 13 & 0.001 \\
& Positive ranks & 1 & \\
$\mathbf{4}$ weeks $-\mathbf{5 2}$ weeks & Ties & 1 & \\
$(n=15)$ & Negative ranks & 5 & 0.322 \\
& Positive ranks & 6 & \\
\hline
\end{tabular}

osteoarthritis) is limited. Two case-series investigating the efficacy of dextrose prolotherapy injections at reducing pain in lateral epicondylitis [29] and chronic groin pain (osteitis pubis and/or adductor tendinopathy) [30] reported mean reductions of 5.3 and 5.0 respectively on $10-\mathrm{cm}$ VAS pain scales. A double-blind randomised controlled trial reported a significant mean pain decrease of 4.6 on a $10-\mathrm{cm}$ pain scale at 16 weeks from baseline [28]. Pain change in the control group was not significant. These results are comparable to our study, where a 4.5point reduction of pain was reported at 18 weeks followup. One study observed patients to long-term follow-up (mean 11.8 months), reporting a mean VAS pain reduction of 5.3 points compared to baseline [30]. Pain reduction was maintained long-term in Ryan et al's study [31], which is similar to the 4-point VAS average pain reduction in our study.

\section{Future studies}

Future studies require more robust methodologies including larger participant numbers, a control group or crossover design, randomisation, and, if possible, blinding to improve the validity of the results. Adverse effects should be explicitly sought to permit a more thorough treatment profile to be compiled.

VAS average pain data from follow-up and one and two weeks post-injection appear of limited application regarding treatment direction, with the decision to reinject patients with the suboptimal symptomatic response coming at four weeks or later. Follow-up at four weeks then monthly may allow better monitoring of the treatment response over the medium- to long-term and facilitate the decision to re-inject for those patients whose symptoms have not responded as well as predicted. 
With some patients requesting a second injection, it may be prudent to administer more than one prolotherapy injection per symptomatic leg, reflecting the protocols of other studies where injections were administered weekly or monthly, ranging from 2 to 12 injections over the study period [29, 32, 33], or until complete resolution of symptoms or no improvements were seen [30,32].

\section{Acknowledgments}

The authors express thanks to the patients, and both the administrative and clinical teams at the London Independent Hospital and Centre for Sports \& Exercise Medicine, Queen Mary, University of London, for their assistance and patience. Special thanks to Dr. Paul Jones and Sister Mel Pritchard for all their help in ensuring the smooth running of the study.

\section{Authors' contributions}

This was a collaborative study. NP and TC contributed to the conception and design of the study, interpretation of the data, as well as drafting and writing of the manuscript, and final approval. MC, OA, and BA collected the data, contributed to manuscript writing. DM and PM contributed to manuscript writing and statistical and Data analysis. OC developed the injection protocol and technique and, contributed to manuscript writing. All authors agree to be accountable for all aspects of the work. All authors read and approved the final manuscript.

\section{Funding}

There are no funding issues. This research received no specific grant from any funding agency in the public, commercial, or not-for-profit sectors. Relevant, registered) have been explained.

\section{Availability of data and materials \\ There are no issues concerning this as strict confidentiality was observed and subjects were anonymised. The authors affirm that this manuscript is an honest, accurate, and transparent account of the study being reported; that no important aspects of the study have been omitted; and that any discrepancies from the study as planned (and, if relevant, registered) have been explained.}

\section{Ethics approval and consent to participate}

Queen Mary, University of London (QMUL) approved this study. QMREC2009/ 22 - A prospective study to assess the effectiveness of prolotherapy in the management of unresponsive and painful MTSS. All subjects provided written informed consent before participating in the study.

\section{Consent for publication}

Participants and QMUL were aware that findings will be disseminated at conferences and results will be published.

\section{Competing interests}

None declared. The authors disclose no conflict or competing of interest, they did not receive any financial payments or other benefits from any commercial entity related to the subject of this article. No outside source of funds was involved in the preparation of or editing of the manuscript.

\section{Author details}

${ }^{1}$ Centre for Sports and Exercise Medicine, William Harvey Research Institute, Barts and The London School of Medicine and Dentistry, Queen Mary, University of London, London, UK. ${ }^{2}$ London SportsCare, London Independent Hospital, London, UK. ${ }^{3}$ European SportsCare, London, UK. ${ }^{4}$ Monash University, Melbourne, Australia.

Received: 5 June 2020 Accepted: 4 February 2021

Published online: 16 April 2021

\section{References}

1. Orava S, Puranen J. Athletes' leg pains. Br J Sports Med. 1979;13:92-7.

2. Kortebein PM, Kaufman KR, Basford JR, et al. Medial tibial stress syndrome. Med Sci Sports Exerc. 2000;32:S27.
3. Yates $B$, White $S$. The incidence and risk factors in the development of medial tibial stress syndrome among naval recruits. Am J Sports Med. 2004; 32:772-80.

4. Winters M, Bakker EWP, Moen MH, Barten CC, Teeuwen R, Weir A. Medial tibial stress syndrome can be diagnosed reliably using history and examination. Br J Sports Med. 2018:52:1-6.

5. Edwards PH, Wright ML, Hartman JF. A practical approach for the differential diagnosis of chronic leg pain in the athlete. Am J Sports Med. 2005:33:1241-9.

6. Moore M. Shin splints. Diagnosis, management, prevention. Postgraduate medicine. 1988:83:199-200, 203-195, 208-110.

7. Moen MH, Tol JL, Weir A, et al. Medial tibial stress syndrome. Sports Med. 2009:39:523-46

8. Mubarak SJ, Gould RN, Lee YF, et al. The medial tibial stress syndrome a cause of shin splints. Am J Sports Med. 1982;10:201-5.

9. Cosca DD, Navazio F. Common problems in endurance athletes. Am Fam Physician. 2007:76:237-44.

10. Newman P, Waddington G, Adam R. Shockwave treatment for medial tibial stress syndrome; a randomized double-blind sham-controlled pilot trial. J Sci Med Sports. 2017 Mar;20(3):220-4.

11. Gomez Garcia S, Ramon Rona S, Gomez Tinoco MC, et al. Shockwave treatment for medial tibial stress syndrome in military cadets: a single-blind randomized controlled trial. Int J Surg. 2017:46:102-9.

12. Smith W, Winn F, Parette R. Comparative study using four modalities in shin splint treatments. J Orthop Sports Phys Ther. 1986;8(2):77-80.

13. Robertson ME. The relative effectiveness of periosteal picking combined with therapeutic ultrasound compared to therapeutic ultrasound in the treatment of medial tibial stress syndrome type II. Faculty of Health at the Durban institute of technology, Durban, South-Africa. http://hdl.handle.net/1 0321/166, https://openscholar.dut.ac.za/bitstream/10321/166/5/Robertson_2 003.pdf.

14. Loudon JK, Dolphino MR. Use of foot orthoses and calf stretching for individuals with medial tibial stress syndrome. Foot \& ankle specialist. 2010; 3:15-20.

15. Winters M, Eskes M, Weir A, Moen MH, Backx FJ, Bakker EW. Treatment of medial tibial stress syndrome: a systematic review. Sports Med. 2013:43(12): 1315-33. https://doi.org/10.1007/s40279-013-0087-0.

16. Yates $B$, Allen MJ, Barnes MR. Outcome of surgical treatment of medial tibial stress syndrome. The Journal of Bone \& Joint Surgery. 2003;85:1974-80.

17. Holder LE, Michael RH. The specific scintigraphic pattern of" shin splints in the lower leg": concise communication. Journal of nuclear medicine. 1984;25:865-9.

18. Michael RH, Holder LE. The soleus syndrome a cause of medial tibial stress (shin splints). Am J Sports Med. 1985;13:87-94.

19. Bhatt R, Lauder I, Finlay D, et al. Correlation of bone scintigraphy and histological findings in medial tibial syndrome. Br J Sports Med. 2000;34:4953.

20. Detmer DE. Chronic shin splints. Sports Med. 1986:3:436-46.

21. Johnell O, Rausing A, Wendeberg B, et al. Morphological bone changes in shin splints. Clin Orthop Relat Res. 1982:167:180-4.

22. Arendt EA, Griffiths HJ. The use of MR imaging in the assessment and clinical management of stress reactions of bone in high-performance athletes. Clin Sports Med. 1997;16:291-306.

23. Beck BR. Tibial stress injuries. Sports Med. 1998:26:265-79.

24. Winters M, Burr DB, van der Hoeven H, Condon KW, Bellemans J, Moen MH. Microcrack-associated bone remodeling is rarely observed in biopsies from athletes with medial tibial stress syndrome. J Bone Miner Metab 2019;37(3): 496-502.

25. Schultz LW. A treatment for subluxation of the temporomandibular joint. J Am Med Assoc. 1937;109:1032-5.

26. Banks AR. A rationale for prolotherapy. Journal of Orthopaedic Medicine. 1991:13.

27. Liu YK, Tipton CM, Matches RD, et al. An in situ study of a Sclerosing solution in rabbit medial collateral ligaments and its junction strength Connect Tissue Res. 1983:11:95-102.

28. Safadi F F, Barbe M F, Abdelmagid S M, et al. Bone structure, development and bone biology. Bone pathology: Springer; 2009. p. 1-50.

29. Scarpone M, Rabago D, Zgierska A, et al. The efficacy of prolotherapy for lateral epicondylosis: a pilot study. Clinical journal of sports medicine. 2008:18:248.

30. Topol GA, Reeves KD, Hassanein KM. Efficacy of dextrose prolotherapy in elite male kicking-sport athletes with chronic groin pain. Arch Phys Med Rehabil. 2005;86:697-702. 
31. Ryan MB, Wong A, Gillies J, et al. Sonographically guided intratendinous injections of hyperosmolar dextrose/lidocaine: a pilot study for the treatment of chronic plantar fasciitis. Br J Sports Med. 2009;43:303-6.

32. Yelland MJ, Sweeting KR, Lyftogt JA, et al. Prolotherapy injections and eccentric loading exercises for painful Achilles tendinosis: a randomised trial. Br J Sports Med. 2011:45:421-8

33. Khan S, Kumar A, Varshney M, et al. Dextrose prolotherapy for recalcitrant coccygodynia. J Orthop Surg. 2008:16.

34. Curtin M, Crisp T, Malliaras P, Padhiar N. The effectiveness of prolotherapy in the management of recalcitrant medial tibial stress syndrome: a pilot study. Br J Sports Med. 2011;45:e1. https://doi.org/10.1136/bjsm.2010.081554.8.

35. Huskisson E. Measurement of pain. Lancet. 1974;304:1127-31.

36. Crossley KM, Bennell KL, Cowan SM, et al. Analysis of outcome measures for persons with patellofemoral pain: which are reliable and valid? Arch Phys Med Rehabil. 2004:85:815-22.

37. Likert R. A technique for the measurement of attitudes. Arch Psychol. 1932.

38. Rompe JD, Cacchio A, Furia JP, et al. Low-energy extracorporeal shock wave therapy as a treatment for medial tibial stress syndrome. Am J Sports Med. 2010;38:125-32

39. Moen MH, Schmikli SL, Weir A, et al. A prospective study on MRI findings and prognostic factors in athletes with MTSS. Scand J Med Sci Sports. 2014; 24(1):204-210

40. Lazzarini KM, Troiano TN, Smith RC. Can running cause the appearance of marrow oedema on MR images of the foot and ankle? Radiology. 1997 Feb; 202(2):540-2.

41. Bergman AG, Fredericson M, Ho C, Matheson GO. Asymptomatic tibial stress reactions: MRI detection and clinical follow-up in distance runners. AJR Am J Roentgenol. 2004 Sep;183(3):635-8.

42. Winters M, Moen MH, Zimmermann WO, Lindeboom R, Weir A, Backx FG, Bakker EW. The medial tibial stress syndrome score: a new patient-reported outcome measure. Br J Sports Med. 2016 Oct;50(19):1192-9.

43. Nauck T, Lohrer H, Padhiar N, King JB. Development and validation of a questionnaire to measure the severity of functional limitations and reduction of sports ability in German-speaking patients with exerciseinduced leg pain. Br J Sports Med. 2015 Jan;49(2):113-7.

44. Korakakis V, Malliaropoulos N, Baliotis K, Papadopoulou S, Padhiar N, Nauck $\mathrm{T}$, Lohrer H. Cross-cultural adaptation and validation of the exercise-induced leg pain questionnaire for English- and Greek-speaking individuals. J Orthop Sports Phys Ther. 2015 Jun;45(6):485-96

\section{Publisher's Note}

Springer Nature remains neutral with regard to jurisdictional claims in published maps and institutional affiliations.

Ready to submit your research? Choose BMC and benefit from:

- fast, convenient online submission

- thorough peer review by experienced researchers in your field

- rapid publication on acceptance

- support for research data, including large and complex data types

- gold Open Access which fosters wider collaboration and increased citations

- maximum visibility for your research: over $100 \mathrm{M}$ website views per year

At $\mathrm{BMC}$, research is always in progress.

Learn more biomedcentral.com/submissions 